\title{
PERIPHERAL EFFECTOR MECHANISM OF GALVANIC SKIN REFLEX
}

\author{
KENTARO TAKAGI AND TERUO NAKAYAMA \\ Department of Physiology, Nagoya University School of Medicine
}

In Tarchanoff's galvanic skin reflex, two portions of human skin are simply connected up with galvanometer. In Veraguth's reflex changes of apparent resistance of the skin is measured by leading a weak current. The former may be called 'endosomatic' or 'potential' and the latter 'exosomatic' or 'current' method $(5,11)$. Both methods have been widely employed, but only a few studies $(1,8,18,21)$ were made on the comparison of these two methods, in which attention was paid chiefly on the one-to-one relationship between the two methods.

On the peripheral mechanism of this reflex, an involvement of sweat gland was already suggested by Tarchanoff (17), which was supported by a considerable number of papers published up to date, although it is not still clear how the sweat glands do manage to produce a GSR. It should be mentioned that discussions on the peripheral mechanism have been made without discriminating the two recording methods. In our previous study (13), the stimulus intensity was varied, the effects of posture, local ischemia and local temperature were observed, and it was concluded that the wave form of GSR in endosomatic method was composed of negative and positive processes. If that be the case, what are physiological changes that give rise to these two processes? The solution of this subject may clarify whether or not the records by two methods indicate an identical physiological event, and furthermore may throw some light on the quantitative treatment of this reflex.

\section{METHODS}

Experimental procedures were the same as previously reported (13). The time constant of amplifier used was 2.5 and $10 \mathrm{sec}$. Upward deflections in records mean an increased negativity of palmar skin (except for fig. 5). For impedance measurement, variable capacitance and resistance were connected in parallel forming one arm of the Wien bridge and alternative current used was sinusoidal wave of 1,000 c.p.s. The balancing of the bridge was checked and photographed simultaneously with GSR on the screen of two beam cathode ray oscillograph. To see the effect of galvanization, direct current was passed through the palmar skin from outside to inside and the circuit was so designed that both the potential and resistance changes were picked up. A value of a

\footnotetext{
Received for publication May 17, 1958.
}

* 高木健太郎, 中山昭雄 
few $\mu A$ was approximately calculated from electric source and resistances of the circuit.

RESULTS

The effect of removal of epidermis. A vesicle was induced with a blistering agent on the left little finger, and the epidermis of $c a .5 \times 5 \mathrm{~mm}$. was taken off by scissors. With this treatment, the outer three layers of epidermis were removed remaining only the stratum Marpighii. The defect of epidermis was covered with saline solution and a small electrode of $2 \times 2 \mathrm{~mm}$. was placed. GSR obtained from this region is shown in fig. $1 \mathrm{~A}$. The lower record of this figure was recorded from the right little finger with electrode of $10 \times 10 \mathrm{~mm}$. After removal of epidermis, painful stimulus was necessary to get response, and the response thus obtained was always positive wave of extremely small amplitude. Negative deflection was never observed. Two days after the removal, regeneration of epidermis was advanced to a some extent. At this stadium, the response became negative wave (fig. $1 B$ upper record) on which only a small positive notch was noticed at its rising phase.

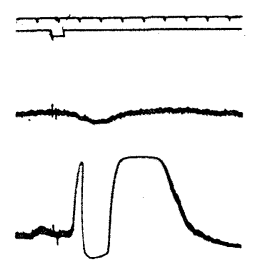

$A$

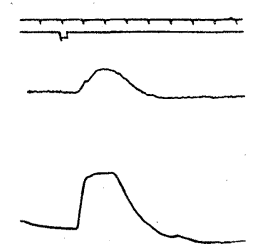

$B$

FIG. 1. The effect of removal of epidermis on Galvanic Skin Reflex. In $A$, upper record was obtained with small electrode on left little finger just after the removal of epidermis. Lower.record was obtained from intact right little finger with large electrode. $B$ was registered two days after the removal of epidermis. Time mark gives 2 sec.

Impedance change during the response. Impedance of palmar skin varies gradually with a lapse of time without any apparent reasons. The upper tracing of fig. 2 is GSR recorded from the left thumb, the time constant of amplifier was $10 \mathrm{sec}$. and the lower tracing is impedance change between the right thumb and the forearm. The skin impedance, though balanced previously, was increased spontaneously to a certain extent before the stimulus was applied. The impedance change continued for longer than a minute in proportion to the long negative wave of response. In all instances, the change of impedance took place in one way; a decrease in resistance and an increase in capacitance. With successive stimulations, negative deflection became smaller and in accordance with this, the duration of impedance change was shortened while the amplitude of positive deflection of GSR was kept constant.

The effect of galvanization. Typical records of endosomatic and exosomatic 


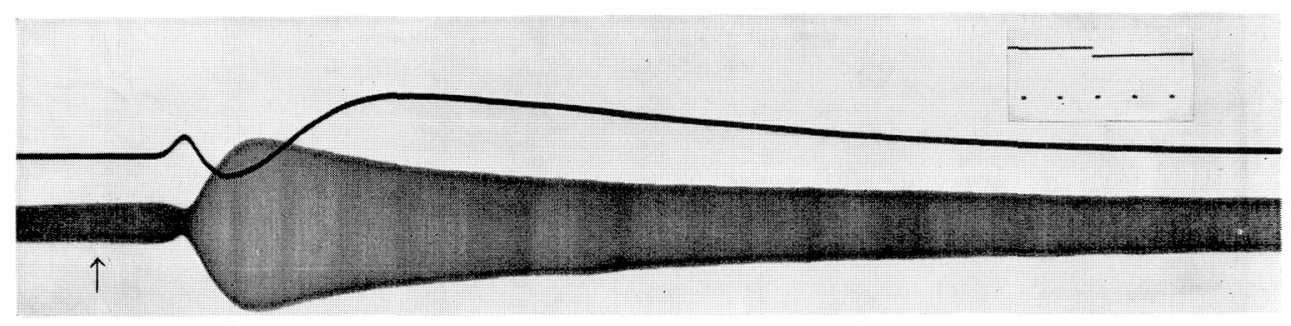

FIG. 2. Simultaneous record of GSR and impedance change. GSR was recorded from left thumb. Impedance change was measured between right thumb and forearm with alternative current of 1,000 c.p.s. Arrow indicates an electric stimulus to the leg. Calibration voltage $1 \mathrm{mV}$, time mark $1 \mathrm{sec}$.

methods are shown in fig. 3. The upper record was obtained by endosomatic method from the left thumb and the lower by exosomatic method from the right thumb with the current flow of $10 \mu \mathrm{A}$ which passed the palmar skin from outside to inside. In contrast to the endosomatic method, where the negative and positive deflections were observed, the record of the exosomatic method was strictly limited to monophasic response. In successive stimulations negative deflection became smaller step by step whilst the positive became larger. In spite of the expectation that, in this series of responses, the reflexes should become larger in succession, the amplitude of exosomatic response became smaller and smaller. The negative deflection of the endosomatic method and the record of exosomatic method paralleled each other in a similar manner.

Fig. $4 A$ was registered in the same condition as fig. 3 , but $4 B$ was obtained after both fingers were tied firmly at their roots by rubber bandage. In the upper record the positive deflection was disappeared by circulatory disturbance and the response became monophasic negative wave, while the lower record

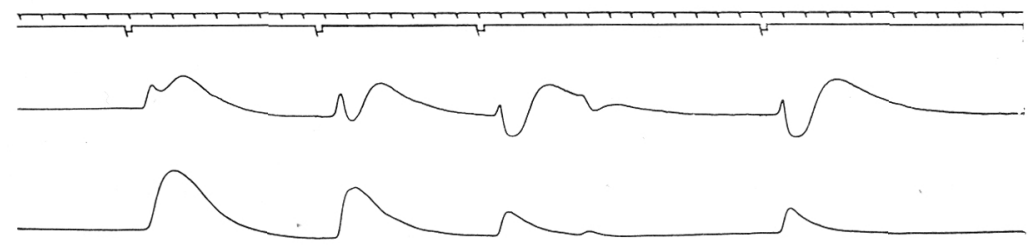

FIG. 3. Comparison of the GSRs obtained by endosomatic (upper, left thumb) and exosomatic methods (lower record, right thumb).
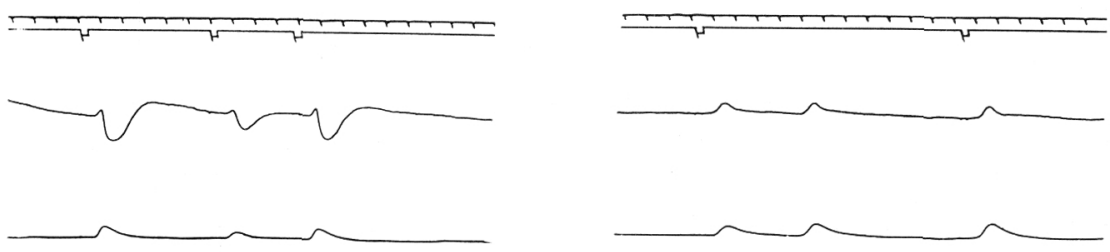

FIG. 4. The effect of local ischemia. Upper (endosomatic, left thumb) lower record (exosomatic method, right thumb). $A$ : control state, $B$ : after local ischemia. 
remained almost unchanged. It can be seen again that the negative deflection and the result by exosomatic method behaved in a similar fashion.

The dependence of the response upon the size of electrode. With use of a small different electrode, an impedance between two electrodes becomes of course larger, so that the response obtained becomes smaller. Are changes of response, if evoked, however, simply those in amplitude? A silver wire, the tip diameter of which was less than $10 \mu$, was inserted with an aid of binocular microscope into sweat droplet on a sweat pore. The responses thus obtained, as illustrated in fig. 5, are monophasic negative waves. Subsequently the electrode was placed on the region other than sweat pores, a very similar response was again attained whilst in the control side the triphasic deflection was recorded with a large electrode.

Upper record of fig. 6 was obtained by a small electrode $(0.8 \mathrm{~mm}$. in diameter $)$ and the lower by a large electrode $(10 \times 10 \mathrm{~mm}$. $)$. Opposed to the above results, positive deflections were more prominent in the use of the small ele-

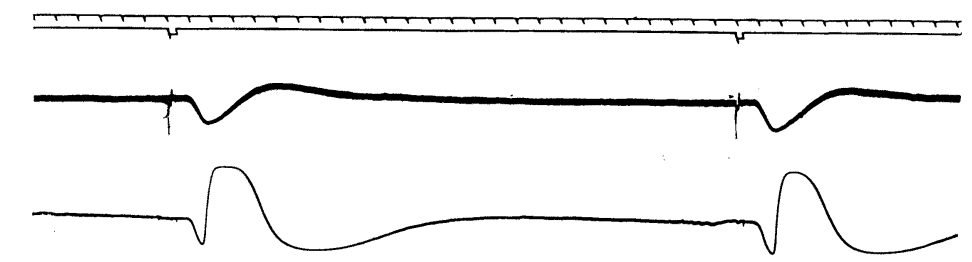

FIG. 5. Upper (left fourth finger) and lower records (left middle finger) were obtained by small silver wire and large plate electrodes respectively. Small electrodes were inserted into sweat pore. Note that downward deflections in this figure mean increased negativity of the palm.

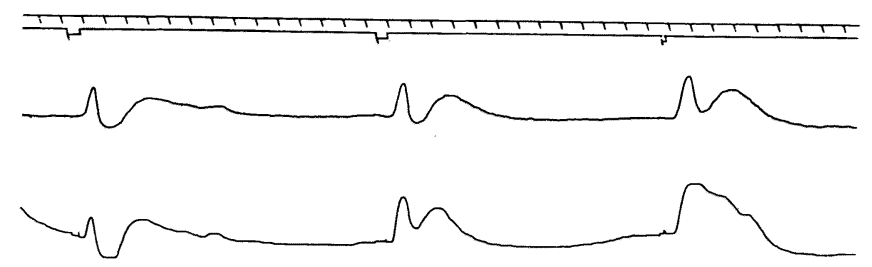

FIG. 6. Upper (left thumb) and lower (right thumb) were obtained by small and large electrodes respectively.

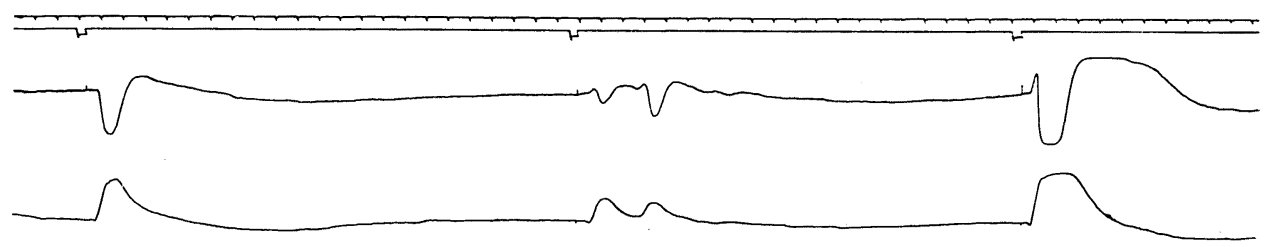

FIG. 7. Upper (right middle finger) and lower records (right fourth finger) were obtained by large and small electrodes respectively. 
ctrode as cleary shown in the second and third responses of this figure. A variety of responses was obtained with small electrodes. For example, as shown in the first responses of fig. 7, upper response which was registered by a large electrode was composed largely of positive deflection whilst the lower response recorded by a small one was of negative deflection, so that it looks like an inverse response at first sight.

\section{DISCUSSION}

The fact that the responses recorded by endosomatic method often shows negative and positive deflections was already described as early as in 1928 as "Mehrphasigkeit des Tarchanoffschen Phänomens," to which an opinion was offered by Gildemeister (7), being based on purely hypothetical scheme. Recently the appearance of positive deflection has been attributed to increased excitability of reflex center (5), and the authors (13) suggested that the response was a summation of two different processes. By the removal of epidermis, in which the most part of total skin resistance lies $(4,12)$, only the positive deflection was obtained and negative deflection was recordable after a slight regeneration of epidermis. With exosomatic method, no GSR could be obtained (4). The impedance change during the response, as above stated, was paralleled in its time course with the negative deflection. As fig. 8 indicates, the

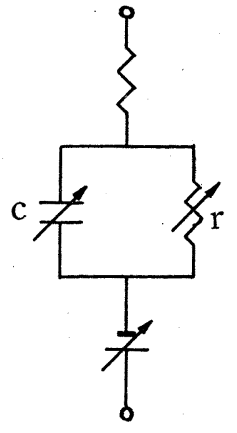

FIG. 8. Fquivalent electric circuit of skin. skin may be considered to be an equivalent electric circuit. Considered in this scheme, amplitude and duration of negative process are dependent on (a) the initial value of $c$ and $r$, (b) the changes of $c$ and $r$ which are proportional to the number of efferent impulses and (c) the voltage and capacity of skin battery which is sustained by local metabolic activity. In the usual exosomatic method, current of $50 \mu \mathrm{A}$ or more is used so that the skin battery is neglected in this case.

The positive deflection, though smaller in size, could be obtained even in the absence of epidermis. It did not parallel with impedance change, and was obtainable less frequently with small electrode. These findings suggest that a physiological process which gives rise to positive deflection is in corium, and it is not of diffuse fashion. It was easily inhibited by local cooling, circulatory disturbance and pressure applied on the same side (13). The most probable source of positive process is sweat gland. Electronegativity evoked by sweat gland activities may be picked up as positive deflection by the existence of skin capacity. Smallness of positive deflection after the removal of epidermis is partly due to injurious effect of blistering agent, but the loss of capacity might be involved in it to a some extent.

An approach to quantitative treatment of GSR has been attempted by several investigators $(3,5,6,9,10)$. The amplitude by itself cannot be an index 
of intensity of response, for the amplitudes of response recorded by exosomatic method, as shown in flg. 3 , became smaller and smaller probably due to decreased resistance level $(2,16)$. On the other hand, the positive deflection which means increased response became larger. To obtain a simple index is. difficult even if various factors which affect the response are estimated simultaneously unless quantitative relationship between them are known. The positive process, the activity of sweat gland, could be an index of reflex, but unfortunately it could not be recorded without accompanying negative one.

The dependence of response on the size of electrode is now recognized easily as follows: There are several sweat glands in a skin area of $1.0 \times 1.0 \mathrm{~mm}$. on the palmar side of finger. With large electrodes, activities of several hundreds of glands are picked up, so that the mean activities are presented as a positive process while in the use of small electrodes the response is largely affected by a few glands just beneath the electrode by chance. With this state of affairs, a formation of complex wave form is expected and indeed this is the case as shown in fig. 9.

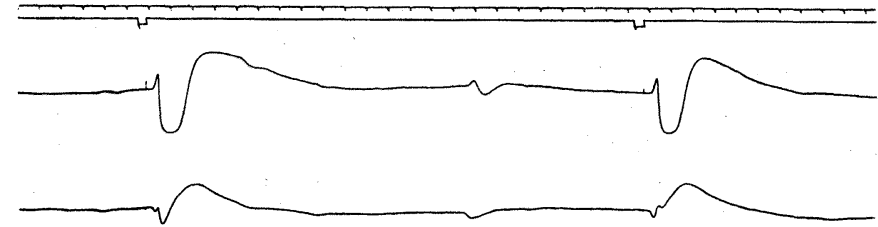

FIG. 9. Upper (right middle finger) and lower records (right fourth finger) were obtained by large and small electrodes respectively.

Local circulatory disturbance could not affect the wave form obtained by exosomatic method while the positive deflection was abolished in endosomatic method. It is inferred, therefore, that the positive process, the function of sweat gland, might be inhibited by such a flow of current which passed through the palmar skin from outside to inside. The mechanism of this inhibition is thought something like anelectrotonic nature. Furthermore this anelectrotonic inhibition of sweat glands may serve to understanding the fact, known empirically, that the current flow is more stable when it passes the skin from outside to inside.

As above stated, negative and positive processes were corresponding to impedance change and sweat glands activities respectively. This does not necessarily mean, however, that the impedance change can occur wholly unrelated with secretory activities. The outbreak of sweat, causing changes in electrolytes in and on the skin, alters the resistance level and may play as a subsidiary factor, but cannot be the essential mechanism of GSR because a similar response was obtained whether the tip of electrode was in sweat droplet or not. 


\section{SUMMARY}

Galvanic skin response was observed in an absence of epidermis, in the existence of ingoing current flow and recorded simultaneously with impedance change. Conclusion was drawn that galvanic skin response recorded by endosomatic method is a summation of two processes: The negative process is caused by change of apparent skin resistance and the positive process is induced by electrical activity of sweat gland.

The variability of wave form with use of small electrode was recognized and a comparison of exosomatic and endosomatic method was made.

\section{ADDENDUM}

In human being, as above stated, polyphasic deflections were often observed with endosomatic method. On the other hand, however, investigations into GSR of animals have shown that response was always monophasic deflection (14, $15,20,22)$. Fixing the animal on the board in any way might exercise an inhibitory action on GSR, because positive deflections are easily suppressed by the pressure applied to the skin (13).

A monkey (macaca fusucata) was held on the author's lap, and electrodes were attached to the palm and forearm to record GSR. Spontaneous fluctuations, similar to the changes in man in hot environment, appeared continuously without any appreciable reasons. In all these and the stimulus (sound) induced response, the waves were all negative deflections. It could not be decided whether the stimulus applied was too weak to evoke positive processes or the properties of sweat glands are different between man and monkey.

\section{REFERENCES}

1. Darrow, C. W. J. exp. Psychol. 10: 197, 1927.

2. DARrow, C. W. J. gen. Psychol. 7: 261, 1932.

3. Darrow, C. W. J. gen. Psychol. 16: 285, 1937.

4. Densham, J. B. AND Wells, J. M. Quart. J. exp. Physiol. 8: 175, 1927.

5. Fuzimori, B. Jap. J. Physiol. 5: 394, 1956.

6. Haggard, E. A. J. exp. Psychol. 35: 46, 1945.

7. Gildemeister, M. Bethe's Handbuch d. norm. u. path. Physiol. V111/2, 783 p. Berlin: Julius Springer, 1928.

8. Jeffress, L. A. J. exp. Psychol. 11: 130, 1928.

9. LACEY, O. L. J. exp. Psychol. 37: 449, 1947.

10. Lacey, O. L. ANd Siegel, P. S. J. exp. Psychol., 39: 122, 1949.

11. MCCleary, R. A. Psychol. Bull., 47: 97, 1950.

12. MCDowall, R. J. Quart. J. exp. Physiol. 23: 277, 1933.

13. Nakayama, T. And TAKAgi, K. Jap. J. Physiol. 8: 21, 1958.

14. Patton, H. D. J. Neurophysiol. 11: 217, 1948.

15. Richter, C. P. AND Whelan, F. J. Neurophysiol. 6: 191, 1943.

16. Seward, J. P. And Seward, G. H. J. exp. Psychol. 18: 64, 1935.

17. TARChanoff, J. Pfiüger Arch. f. d. ges. Physiol. 46: 46, 1890.

18. Thouless, R. H. Brit. J. Psychol. 20: 219, 1930.

19. Veraguth, O. Das psycho-galvanische Reflexphänomen. Berlin: Karger, 1909.

20. WAng, G. H. And LU, T. W. Chin. J. Physiol. 4: 393, 405, 1930.

21. Wells, F. L. AND Forbes, A. Arch. Psychol. N. Y. 16: 1, 1911.

22. Yokota, T. J. Physiol. Soc. Japan 19: 724 p., 1957. 